> De l'expérience et de la parole des malades, il est toujours plus question à mesure que s'est développée une médecine scientifique pouvant s'en dispenser. Divers lieux et pratiques sont récemment apparus pour faire entendre leur plainte : comités d'éthiques, groupes de paroles, soins palliatifs, comités d'examen des plaintes, notamment. De multiples façons, le malade est aujourd'hui invité à témoigner de son expérience, à exprimer le bouleversement vécu et à faire connaître ses besoins. Trois expériences de la parole sont examinées ici, du point de vue du malade et de leur signification culturelle : la douleur, la souffrance et la considération. Ce sont trois demandes adressées aux soignants, mais aussi trois expériences par lesquelles le malade est amené à penser son individualité et sa relation aux autres, à faire retour sur son passé et à réfléchir sur ce qu'il est et aspire à être. L'expérience de la maladie est ainsi devenue un moment privilégié de la construction de l'identité individuelle. <

\section{La douleur}

Depuis le xVIII e siècle, au moins, l'homme occidental porte une attention toujours croissante aux sensations organiques, devenues l'une des source de sa sensibilité et de la manière dont il réfléchit son existence. La perception des sensations internes et l'attention qu'il leur prête a été l'un des moyens par lesquels il a pensé son unité [1], s'est reconnu une intériorité et a médité à la fois son indépendance à l'égard du monde extérieur et son appartenance à la nature.

La perception interne du corps propre, la cénesthésie, la manière dont l'âme est informée de l'état du corps, a connu différentes formes. Jusqu'au XIXe siècle, une approche sensualiste a interprété la personnalité psychique comme le résultat des sensations organiques et

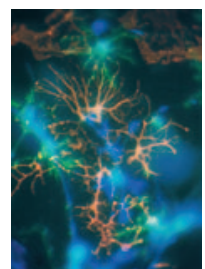

des mouvements du corps. La psychanalyse inversera en partie le schéma, faisant du corps l'expression des désirs et des conflits psychiques; inversion partielle, puisque les pulsions conservent chez Freud une détermination centrale. La sociologie, en parallèle, interprétera le corps comme l'effet d'un dressage, notamment par le langage qui module l'expression corporelle et les sensations pour en faire des expériences normées et intelligibles [2]. Le corps est ainsi devenu un objet privilégié de réflexion sur l'individualité et l'universalité, ce qui nous fait semblables et différents, identiques et uniques à la fois. La plainte du malade porte largement sur la menace intérieure et extérieure pesant sur lui. La douleur dessine une frontière, celle de son identité.

Claudine Herzlich [3] a montré comment la maladie, dans ses représentations profanes, pouvait être associée au monde extérieur qui agresse l'individu: pollution, bactéries, virus, stress, dont la société est responsable par le mode de vie malsain qu'elle impose. La maladie est extérieure et l'individu doit s'en préserver, conserver sa santé. La santé publique, par ses discours et interventions, légitime en partie cette représentation. Que la maladie soit vécue comme destructrice, un événement qui désorganise la vie, qui oblige à rompre avec ses activités ordinaires, à suspendre ses responsabilités, qu'elle soit vécue, au contraire, comme libératrice, une occasion de rompre avec le cours ordinaire de l'existence, de changer sa vie, de se connaître et d'en sortir grandi, ou qu'elle 
soit encore vécue comme un métier, avec la maladie chronique, une condition qui appelle une réorganisation permanente de son existence, elle est toujours le moyen pour l'individu de se saisir. II s'agit toujours de penser l'individu, dans sa singularité, son intégrité et sa singularité. La personnalisation de la mort dans les soins palliatifs en fournit un autre exemple: une expérience ménagée par des experts, qui donne toute la place à l'expérience singulière de la mort, et qui est temps de réflexion sur soi. La douleur et la souffrance deviennent le premier objet d'intervention, avec le développement d'une spécialité médicale qui les explique autant par des facteurs biologiques qu'émotionnels et sociaux (exemple: déni et peur de la mort; conflits familiaux non résolus) $[4,5]$.

Le corps est devenu central dans l'expérience contemporaine, et la plainte du malade contient une interrogation et une inquiétude sur son identité. II a acquis une certaine évidence : il est la première certitude de l'individu, ce qui assure et exprime son individualité, sa réalité, pourrions-nous dire. Mais cette matérialité du corps, loin de les congédier, confère une place toujours plus grande au langage et à la parole. L'individualisation du corps et de l'identité oblige chacun à s'interpréter, se décrire et se raconter, et la société propose de multiples savoirs, instruments et lieux d'expression pour aider l'individu à le faire (livres, tests, témoignages, auto-examen de l'âme, du corps et de son mode de vie...).

\section{La souffrance}

Dans la mesure où la douleur est déjà la mise en mots des sensations corporelles [6], elle conduit rapidement à la souffrance, qui est une expérience plus radicale encore, celle de la perte de la parole. La souffrance est le plus fortement ressentie par ces individus blessés, traumatisés, enfermés dans leur mutisme, à la suite d'une violence physique, psychologique ou morale, et qui ne trouvent plus les mots [7]. La maladie grave conduit également parfois à cette expérience de l'indicible, à ce sentiment qu'il est très difficile de se faire entendre, que la parole est impossible. Cancer, maladies dégénératives et lourds handicaps mettent à l'épreuve la parole, ils demandent à être racontés, mais laissent sans voix. Expériences du corps, là encore, et indissociablement, expériences du langage. La souffrance est ce besoin pressant de raconter le bouleversement vécu, et le sentiment que la parole est vaine, qu'il n'y a pas de mots pour le dire de façon adéquate et être compris.

La possibilité de mettre son expérience en récit et de le faire entendre devient centrale dans la relation thérapeutique et la manière dont la maladie est vécue $[8,9]$. Celle-ci est d'ailleurs souvent vécue et représentée comme communication entravée, impossibilité de se faire entendre [10]. Ce défaut de communication est conséquence de la maladie, parce que la maladie isole, suspend et compromet les relations sociales, interrompt les activités; il appelle du même coup le rétablis- sement de la parole sans laquelle on demeure à l'écart, dans la maladie ou l'anormalité. La communication entravée est aussi expression de la maladie, sinon la maladie elle-même, en ce sens que c'est ce défaut de communication qui fait souffrir, en plus de la douleur physique; que les autres ne peuvent pas ou ne veulent pas savoir et comprendre les angoisses ou les peurs du malade le font souffrir. Enfin, pour plusieurs, elle est même perçue comme source de la maladie, ainsi que de nombreuses théories et de conceptions profanes ou scientifiques le laissent entendre de nos jours à la suite de la psychosomatique et de la psychanalyse : ce qui devait être dit ne l'a pas été, les difficultés avec l'autre ont été refoulées, sont restées non dites, les émotions et les frustrations sont demeurées silencieuses [11]. L'indicible est cette rupture de sens [11-13]. II est sentiment d'incompréhension de ce qui arrive et de l'impossibilité de le dire et d'être entendu. La souffrance est une expérience de la parole, et donc de notre rapport aux autres, de ce qu'ils peuvent comprendre et nous répondre. Elle appelle le silence et la parole tout à la fois. Elle est manifestation et approfondissement de cette subjectivité, non plus à partir de l'unité du moi comme pour la douleur, mais de la relation à l'autre. Elle est le sentiment, provoqué par la maladie, d'un écart s'élargissant, d'une distance et d'une rupture de la conversation, du cours ordinaire de la vie. La souffrance, c'est à la fois la nécessité et la difficulté ressenties de faire le récit, de donner un sens à un flot d'émotions et de sensations, pour les comprendre et ne plus simplement les subir; un récit que l'incompréhension, la honte ou la peur empêchent d'exprimer [7]. «La vie n'est pas une histoire, écrit Boris Cyrulnik. C'est une résolution incessante de problèmes d'adaptation. Mais la vie humaine, elle, nous contraint à en faire une histoire pour éviter de la réduire à une série de réactions de défense pour la survie » [14]. Cette histoire permet de sortir du présent et d'ouvrir un avenir, en ouvrant la parole, car on a besoin de la réponse des autres pour discerner ce qui est vrai, juste ou désirable. L'expérience doit être nommée et comprise par les autres, autrement elle demeure indéfinie et prive l'existence d'unité et de direction.

Les groupes de parole sont l'un des lieux où la souffrance trouve à s'exprimer. Réunissant des personnes souffrant d'une maladie grave, mortelle ou invalidante (cancer, maladie mentale, handicap), ils permettent l'échange de conseils et de soutien, mais également le récit des expériences. D’avoir reçu un diagnostic ou un pronostic semblable, subi les mêmes traitements, affronté la même angoisse, ressentant une douleur identique, fait en sorte que chacun pressent que les autres pourront saisir le sens des mots utilisés et la signification des comportements relatés. L'expérience commune atténue les ambiguïtés du langage, lève les doutes. Les individus ont ainsi la possibilité d'affirmer à la fois leur singularité et le caractère commun de leur expérience [10, $11,15]$. Dans ces espaces abrités, en marge des liens sociaux normaux, l'expérience peut être racontée et les silences sont autorisés. Le récit devient possible [10]. 
La forte sensibilité contemporaine à la souffrance n'est pas étrangère à celle pour la communication et la parole. Elle traduit une inquiétude dans la confiance que l'on peut avoir envers les mots et envers les autres. Elle est le sentiment de la précarité de notre existence, mais aussi de la fragilité de nos liens et de la parole. La souffrance, c'est l'expérience de la perte d'un sens commun et donc de la possibilité de communiquer. Une expérience de la singularité à nouveau, mais aussi de la solitude.

\section{La considération}

Alain Ehrenberg [16] a attiré l'attention sur une souffrance plus largement répandue, plus diffuse également, la dépression. Il associe l'importance qu'elle a prise aux transformations de l'identité et de l'individualité dans la société contemporaine. Devenue l'une des principales expressions du malheur intime, elle est le signe de l'épuisement, de l'insécurité et de l'incapacité des individus devant l'obligation qui leur est faite d'être performant, de se dépasser, de se réaliser, de trouver leur propre voix. L'accomplissement oblige à redéfinir sans cesse son identité et à obtenir l'approbation ou la reconnaissance des autres des choix que l'on a fait, de la vie que l'on mène, de ce que l'on cherche à être. Elle est la maladie de l'individu libre et laissé à lui-même, et dont la quête est génératrice d'anxiété et de frustration.

Marquant une rupture de la parole moins grande que la souffrance dont nous avons précédemment parlé, cette plainte témoigne d'une demande de plus en plus étendue de reconnaissance et de considération. La société démocratique laisse davantage l'individu libre de son destin, avec l'angoisse et l'incertitude qui accompagnent cette liberté, tout en prônant une plus grande égalité : chacun a droit à la même attention, un égal respect, aux mêmes chances $[17,18]$.

Cette demande de considération, on la retrouve dans les plaintes ou insatisfactions exprimées par les usagers pour les services reçus, qu'elles portent sur la qualité de l'acte professionnel posé (erreur de diagnostic), l'accessibilité à des services (peu d'aide reçue à domicile par une personne handicapée), le comportement des soignants (impolitesse, manque d'écoute, inconduites) ou encore les frais déboursés, par delà les questions spécifiques que chacune de ces plaintes soulèvent. On ne se sent pas écouté, pris au sérieux, digne du respect, de l'attention et des soins qu'une personne devrait recevoir [19]. L'erreur ou la négligence témoignent du peu de valeur que l'on se voit accorder. Cette plainte s'exprime dans une demande de réparation: excuses, reconnaissance de la faute, désir d'être entendu, demande pour recevoir certains services. Elle se prolonge même parfois dans l'insatisfaction à propos de la manière dont la plainte a été traitée... Se plaindre des soins et des services reçus, c'est protester contre l'image que les autres semblent avoir de soi.
Cette demande de considération ne prend pas uniquement la forme d'une demande individuelle. Elle est au cœur des revendications des groupes et associations de malades ou handicapés, pour obtenir des droits ou des services, participer aux décisions, changer la représentation de la pathologie, sortir les malades du silence et de l'indifférence des autres. Elle trouve à s'exprimer dans divers lieux où la parole de l'usager est sollicitée : comités d'usagers, comités d'éthique, consultations publiques sur les services de santé... [15, 18, 20]. Elle trouve écho auprès des administrations, qui l'enregistrent et la comptabilisent au profit de la gestion des établissements et des services, comme un indicateur des besoins ou de la qualité des services [19].

À travers la formulation de sa plainte ou de ses plaintes s'expriment une recherche et une demande qui dépassent largement la maladie et même le corps, et qui concernent l'ensemble du rapport à soi et aux autres $[7,21]$. Cette troisième figure de la plainte touche elle aussi l'identité. Elle pose la question de savoir ce que l'on vaut et ce que l'on aspire à devenir, la représentation que l'on se fait d'une vie digne et valable et de la difficulté de s'en approcher, particulièrement lorsque les formes d'accomplissement et de reconnaissance sont fragiles (famille, travail, conjugalité, consommation). L'attention à l'estime de soi, les multiples formes d'accompagnement et de soutien (psychotropes) sont des réponses à cette plainte. Dans un monde où son identité n'est jamais acquise, ce sont des moyens pour s'assurer de sa propre existence [16].

\section{Expérience et culture}

D'autres figures seraient sans doute discernables. Douleur, souffrance et considération ne représentent pas des catégories de patients, mais différentes demandes. Un même patient en exprimera deux ou les trois, successivement ou simultanément. La plainte du malade, on l'a souligné maintes fois, est l'expression d'expériences et de demandes nombreuses et variées $[6,7,12]$. D'autres définitions et interprétations de la souffrance et de la douleur sont bien sûr possibles; c'est au regard du rapport à soi et aux autres, et des significations culturelles, qu'elles sont ici comprises.

Ces trois expériences ne sont pas propres au monde contemporain, qui en ont cependant donné des figures particulières, et les ont approfondies. L'importance qu'elles prennent, et qui se manifeste à travers la demande toujours croissante de soins, de médicaments et d'espaces d'écoute, est liée à des transformations de la culture. Un profond mouvement d'égalisation des conditions et des statuts dans les sociétés démocratiques est à l'œuvre, qui appelle cette parole que sollicitent les associations et les médias, qu'enregistrent les administrations et qu'interprètent les soignants $[15,20]$. Une profonde transformation de la subjectivité est simultanément à l'œuvre, une individualisation de l'expérience, et la médecine scientifique, loin de toujours y faire obstacle, contribue à la renforcer, en centrant l'attention 
sur le corps justement, devenu expression première de la singularité et de la subjectivité. Loin d'être simplement une réaction à une déshumanisation de la médecine, ces expériences ont pris de l'importance à mesure que s'est développée une médecine scientifique $[22,23]$. Dans la parole des usagers, on distingue diverses plaintes, à travers lesquelles s'expriment différentes demandes, que l'on n'entendait pas, ou pas autant, auparavant. Ces transformations appellent d'ailleurs une demande de services, de consultations, d'interventions, d'accompagnement et de techniques qui soutiennent ce travail sur soi.

Dans une culture où les conduites naturelles, la vie normale ou désirable et les vérités indiscutables sont de moins en moins évidentes, le corps devient le lieu de sa certitude et de son identité. Le corps a en effet quelque chose d'évident, de tangible. Si le corps est muet, le malade le fait parler à la manière d'une poupée ventriloque, en projetant sur lui un discours fait de valeurs, de normes et de vérités. Parlant de sa maladie, le malade parle d'autres choses. Et il parle plus que jamais. $\diamond$

\section{SUMMARY}

Facets of patient complaints: pain, suffering, and the importance of consideration

Patient complaints, as it has often been recognized, serve to express multiple and varied experiences and needs. A number of options for filing complaints and a variety of ways for doing so have arisen recently, among which are ethics committees, support groups, palliative care units, and complaint examination committees. Today patients have many occasions for expressing their experiences, talking about distressing incidents, and making their needs known. Three orally related experiences are examined here, both from the viewpoint of the patient and in light of their cultural meanings: pain, suffering, and the importance of consideration. These are three expectations of care providers, but also three experiences that dispose patients to rethink their individuality and their relationships with others, to take stock of their pasts, and to reflect on who they are and who they want to become. The experience of illness has thus become pivotal in the process of constructing individual identity. $\diamond$

\section{RÉFÉRENCES}

1. Azouvi F. Quelques jalons dans la préhistoire des sensations internes. Collection Revue de synthèse. Paris : Albin Michel, 1984 ; 113-114 : 113-33.

2. Starobinski J. Brève histoire de la conscience du corps. In : Ellrodt R, ed. Genèse de la conscience moderne. Paris : PUF, 1983 : 215-29.

3. Herzlich C. Santé et maladie. Analyse d'une représentation sociale. Paris: Mouton, $1969: 210 \mathrm{p}$

4. Moulin P. Les soins palliatifs en France : un mouvement paradoxal de médicalisation du mourir contemporain. Cahiers Internationaux de Sociologie 2000 ; CVIII : 125-59.

5. Castra M. Bien mourir. Sociologie des soins palliatifs. Paris : PUF, $2003: 366$ p.

6. Benoist J. Illnes, disease, sickness. La Recherche 1995; 281 (suppl) : 7-9.

7. Ricœur P. La souffrance n'est pas la douleur. Autrement 1994 ; $142: 58-69$.

8. Pierret J. The illness experience: state of knowledge and perspectives for research. Sociology of Health and Illness $2003 ; 25$ (silver anniversary issue) : 4-12.

9. Kleinman A. Writing at the margin. Discourse between anthropology and medicine. Berkeley : University of California Press, 1995 : 314 p.

10. Gagnon $\varepsilon$. La communication, l'autre, l'indicible. De l'entraide des malades. Anthropologie et Sociétés $1999 ; 23: 61-78$.

11. Paquet S. Folie, entraide et souffrance. Paris: L'Harmattan, $2001: 134$ p.

12. Good BJ. Medecine, rationality and experience. An anthropological perspective. New York: Cambridge University Press, 1994 : 242 p.

13. Kleinman A, Das V, Lock M. Social suffering. Berkeley: University of California Press, $1997: 404 \mathrm{p}$.

14. Cyrulnik B. In : Un merveilleux malheur. Paris : Odile Jacob, 2002 : 113-4.

15. Broqua P. Jauffret-Routide M. Les collectifs d'usagers dans les champs du sida et de la toxicomanie. Med Sci (Paris) $2004 ; 20: 475-9$.

16. Ehrenberg A. La fatigue d'être soi. Paris: Odile Jacob, $1998: 414 \mathrm{p}$.

17. Taylor C. Le malaise de la modernité. Paris : Cerf, $1994: 150 \mathrm{p}$.

18. Fassin D. Des maux indicibles. Sociologie des lieux d'écoute. Paris: La Découverte, $2003: 198 \mathrm{p}$.

19. Clément M. Gagnon $\varepsilon$. La rencontre avec l'usager comme sujet de droit : la mise en scène de sa parole dans le régime d'examen des plaintes. In : Cresson G, Schweyer F, eds. Valeurs et normes dans le champs de la santé. Rennes : Éditions de l'École Nationale de Santé Publique, 2004 : 291-301.

20. Gagnon $\varepsilon$, Clément M. La parole de l'usager. In : Saillant F, Clément M, Gaucher C, eds. Identités, vulnérabilité, communautés. Québec: Nota Bene, 2004 : 85-103.

21. Fassin D. Quand le corps fait loi. La raison humanitaire dans les procédures de régularisation des étrangers. Sciences Sociales et Santé $2001 ; 19: 5$-32.

22. Baszanger I. Douleur, travail médical et expérience de la maladie. Sciences Sociales et Santé 1989 ; VII : 5-34.

23. Rey R. Avec ou sans douleur : une conquête dans l'Histoire. Autrement 1994 ; 142 : $72-84$.
TIRÉS À PART

દ. Gagnon 